\title{
LncRNA STXBP5-AS1 suppresses stem cell-like properties of pancreatic cancer by epigenetically inhibiting neighboring androglobin gene expression
}

Shi Chen ${ }^{1 *}$, Long Huang ${ }^{1 \dagger}$, Ge Li ${ }^{2 \dagger}$, Funan Qiu' ${ }^{1}$ Yaodong Wang ${ }^{1}$, Can Yang ${ }^{1}$, Jingjing Pan ${ }^{1}$, Zhangwei Wu ${ }^{1}$, Jiangzhi Chen ${ }^{2^{*}}$ and Yifeng Tian ${ }^{1^{*}}$ (D)

\begin{abstract}
Previous studies suggest the tumor suppressor role of long non-coding RNA (InCRNA) STXBP5-AS1 in cervical and gastric cancer, but its expression pattern and functional mechanism are still elusive in pancreatic cancer (PC). Relative expression of STXBP5-AS1 in PC both in vivo and in vitro was analyzed by real-time PCR. IC $C_{50}$ of Gemcitabine was determined by the MTT assay. Cell proliferation in response to drug treatment was investigated by colony formation assay. Cell apoptosis was measured by both caspase-3 activity and Annexin V/PI staining. Cell invasion capacity was scored by the transwell assay in vitro, and lung metastasis was examined with the tail vein injection assay. Cell stemness was determined in vitro by sphere formation and marker profiling, respectively, and in vivo by limited dilution of xenograft tumor incidence. Subcellular localization of STXBP5-AS1 was analyzed with fractionation PCR. Association between STXBP5-AS1 and EZH2 was investigated by RNA-immunoprecipitation. The binding of EZH2 on ADGB promoter was analyzed by chromatin immunoprecipitation. The methylation was quantified by bisulfite sequencing. We showed downregulation of STXBP5-AS1 in PC associated with poor prognosis. Ectopic STXBP5-AS1 inhibited chemoresistance and metastasis of PC cells. In addition, STXBP5-AS1 compromised stemness of PC cells. Mechanistically, STXBP5-AS1 potently recruited $E Z H 2$ and epigenetically regulated neighboring $A D G B$ transcription, which predominantly mediated the inhibitory effects of STXBP5-AS1 on stem cell-like properties of PC cells. Our study highlights the importance of the STXBP5-EZH2-ADGB axis in chemoresistance and stem cell-like properties of PC.
\end{abstract}

Keywords: Long non-coding RNA, STXBP5-AS1, ADGB, Pancreatic cancer

*Correspondence: wawljwalj@126.com; zeysson1@126.com; tianyifengs|@126.com

†Shi Chen, Long Huang, and Ge Li have contributed equally to this work ${ }^{1}$ Department of Hepato-Biliary-Pancreatic Surgery, Fujian Provincial Hospital, Shengli Clinical Medical College of Fujian Medical University, Fujian Medical University, No. 134 East Street, Fuzhou 350001, Fujian, China

2 Department of Hepatobiliary Surgery, Union Hospital, Fujian Medical University, Fuzhou 350001, Fujian, China

\section{Background}

Pancreatic cancer (PC) is one of the most lethal human malignancies with poor prognosis [1]. There are 227,000 deaths claimed by this disease every year globally. Currently, surgical removal and chemotherapy are still the mainstay of clinical management options [2]. However, due to the lack of evident symptom and reliable early diagnosis, PC is frequently diagnosed at late and untreatable stage, which greatly contributes to the relatively unfavorable prognosis $[3,4]$. On the other hand, assembled studies suggest the existence of specific 
subpopulation in pancreatic tumor cells with characteristic features of self-renewal, differentiation and capability of driving tumor incidence and metastasis [5-7]. More importantly, the stem cell-like tumor cells are deemed as the major cause of resistance to both conventional chemotherapy and radiotherapy [8-10]. Therefore, insightful understanding into pathogenesis of PC and characterization of tumor stem cells involved in this disease is extremely critical in the search for early diagnostic marker and therapeutic targets.

Long non-coding RNA (lncRNA) is a class of RNA molecules with an average length of more than $200 \mathrm{nt}$ and no protein coding potential [11]. Increasing evidences support the fundamental roles of lncRNA in multiple aspects of tumor biology in almost all human cancer types, including cell differentiation, proliferation, apoptosis, metastasis and cell stemness [12-15]. More recent investigations suggest the association between lncRNAs and $E Z H 2$, which lead to assembly of polycomb repressive complex 2 (PRC2) and enhance local histone H3 lysine 27 methylation, and consequently inhibited target gene expression epigenetically [16-18]. Here, we focused on a novel lncRNA, STXBP5-AS1, in PC, which was previously investigated in cervical and gastric cancers. Huang et al. first reported that STXBP5-AS1 suppressed cell proliferation, invasion and migration through blockading the PI3K/AKT pathway, which was predominantly mediated by negative regulation on STXBP5 expression in nonsmall-cell lung carcinoma [19]. Subsequently, Cen et al. confirmed the involvement of STXBP5-AS1/PI3K/AKT in tumor suppressive effects in gastric cancer [20]. While in cervical cancer, Shao et al. suggested that STXBP5-AS1 functioned as a competing endogenous RNA to upregulate PTEN via sponging miR-96-5p to reduce cervical cancer cell proliferation and invasion [21]. Notably, Ham et al. found that both ginsenoside Rg3 and Korean red ginseng extracts were capable of epigenetically regulating the expression of tumor-related RFX3-AS1 and STXBP5$A S 1$ [22], therefore providing experimental evidences in support of the targetability of STXBP5-AS1 by traditional Chinese medicine. Our study evidenced the anti-tumoral properties of STXBP5-AS1 in PC, suppression of which rendered drug resistance and stem cell-like features to PC cells. We further demonstrated the epigenetic regulation of $A D G B$ by $S T X B P 5-A S 1$ via interacting with and potently recruiting $E Z H 2$. Therefore, our data highlighted the critical contributions of STXBP5-AS1 in PC.

\section{Materials and methods Clinical samples}

A total of 60 PC tumors with paired adjacent normal tissues were collected from Fujian Provincial Hospital, Shengli Clinical Medical College of Fujian Medical
University, Fujian Medical University. Written consents were obtained from all enrolled patients, and approval from the Institutional Ethics Committee was received before initiation of this study. Diagnosis was confirmed by independent pathologists, and the specimens were immediately flash-frozen in liquid nitrogen.

\section{Cell culture and treatment}

PC cell lines (AsPC-1, SW1990, Capan-2, CFPAC-1, PANC-1 and Mia PaCa-2) and the normal human pancreatic ductal cell line hTERT-HPNE were ordered from the American Type Culture Collection (ATCC, VA, USA). All cancer cells were maintained in RPMI-1640 (Sigma, MO, USA) containing 10\% fetal bovine serum (Invitrogen, CA, USA) and 1\% antibiotics (penicillinstreptomycin, Hyclone, MA, USA). The hTERT-HPNE cells were cultured following the ATCC recommendation in $75 \%$ glucose-free DMEM (supplemented with L-glutamine and sodium bicarbonate, Sigma, MO, USA) and 25\% M3 Base Medium (Incell, CA, USA). 5-Aza-CdR was purchased from Sigma (St. Louis, MO, USA), and cells were treated with the optimal concentration of 5-Aza$\mathrm{CdR}(1 \mu \mathrm{M})$ for $72 \mathrm{~h}$. Regular cell culture was performed in humidified $\mathrm{CO}_{2}(5 \%)$ incubator at $37^{\circ} \mathrm{C}$.

\section{Gene overexpression and knockdown}

STXBP5-AS1 and ADGB overexpression cell lines were established by infecting cells with lentivirus containing the STXBP5-AS1 (pSIN-STXBP5-AS1) and ADGB sequences (pSIN-ADGB), followed by puromycin selection to acquire stable overexpression cells. STXBP5-AS1 and $E Z H 2$ knockdown was achieved by transfecting siRNAs of the following sequences using Lipofectamine 2000 (Thermo Fisher Scientific) according to the manufacturer's instructions:

si-STXBP5-AS1-1: GCAAGTTGCTGAGTATTAT. si-STXBP5-AS1-2: GGATCTTATTCTCCCACAT. si-EZH2-1: GGTGAATGCCCTTGGTCAATA. si-EZH2-2: GAAGCAAATTCTCGGTGTCAA.

\section{Real-time PCR}

RNA was extracted with the TRIzol Reagent (Invitrogen, MA, USA) in accordance with the manufacturer's manual. cDNA synthesis was conducted with $1 \mu \mathrm{g}$ of RNA with cDNA Synthesis Kit (Takara, Ohtsu, Japan). Relative mRNA was quantified with SYBR Premix Ex Taq (TaKaRa) on Applied Biosystems 7900 PCR System (Applied Biosystems, CA, USA). The quantification of gene level was calculated by the $2^{-\Delta \Delta C T}$ method, using GAPDH as the internal reference gene. The primer sequences were listed as below:

STXBP5-AS1 F: 5'-AGGGACTTGCCTTGTCGC TGAT-3'; 
STXBP5-AS1 R: 5'-GAGATTTAGGTGGGGACG CTGC-3';

GAPDH F: 5'-ACGGATTTGGTCGTATTGGGCG-3'; GAPDH R: $5^{\prime}$-GCTCCTGGAAGATGGTGATGGG-3'; Sox2 F: 5'-TGCACCGCTACGACGTGAGC-3'; Sox2 R: 5'-GCCCTGGAGTGGGAGGAAGA-3'; Bmi1 F: $5^{\prime}$-GCTTCAAGATGGCCGCTTG-3'; Bmi1 R: 5'-TTCTCGTTGTTCGATGCATTTC-3'; Lin28 F: 5'-AAAGGAGACAGGTGCTAC-3'; Lin28 R: $5^{\prime}$-ATATGGCTGATGCTCTGG-3'; Nanog F: 5'-AGTTGGACAGGGAGATGGC-3'; Nanog R: 5'-AACCTTCCTTGCTTCCACG-3'; ADGB F: 5'-AGACCCTCATCAGAAGTGCAG-3'; ADGB R: 5'-GCTACCAGAGGACAAGACCTACT-3'.

\section{Sphere formation assay}

400 cells were seeded into 6 -well plate, and 50 cells were seeded into 24-well plate, followed by continuous culture for 10 days. Spheres were maintained in serum-free DMEM/F12 medium containing 2\% B27 (Invitrogen, MA, USA) plus EGF (20 ng/ml), bFGF $(20 \mathrm{ng} / \mathrm{ml})$ and insulin $(5 \mu \mathrm{g} / \mathrm{ml}$ from PeproTech, NJ, USA).

\section{Cell viability and apoptosis}

The indicated cells were prepared in 96-well plate $\left(10^{4}\right.$ cells/well) and treated with serial concentrations of Gemcitabine. After $48 \mathrm{~h}$, cell viability was monitored by the MTT assay, and $\mathrm{IC}_{50}$ value of Gemcitabine was determined with SPSS 23 . To measure cell apoptosis, the indicated cells were treated with $100 \mathrm{ng} / \mathrm{ml}$ of Gemcitabine. After $48 \mathrm{~h}$, single-cell suspension was prepared and stained with Annexin V-FITC-PI Apoptosis Detection Kit (Sigma, MO, USA) as suggested by the provider, and followed by FACS analysis on CytoFlex (Beckman Coulter, CA, USA).

\section{Colony formation assay}

Well-dispersed single cells were seeded into 6-well plate (500 cells/well) and subjected to drug treatment for $48 \mathrm{~h}$ at $37^{\circ} \mathrm{C}$. Fresh medium was then replaced, followed by consecutive culture for another 10 days. Colonies were fixed with $3 \%$ formaldehyde briefly and stained with $0.5 \%$ crystal violet for 15 min (Sigma, MO, USA).

\section{Transwell assay}

Invasion capacity was assessed using the transwell chamber which was pre-coated with $1 \%$ Matrigel (BD Biosciences, CA, USA). Cells $\left(10^{3} /\right.$ well $)$ were seeded into insert and cultured in serum-free medium. The lower compartment was supplied with complete medium as chemo-attractant. After $12 \mathrm{~h}$, the non-invaded cells were washed off and invaded cells were fixed with cold-methanol and stained with $0.25 \%$ crystal violet.

\section{Lung colonization model}

PANC-1 cells (either vector control or STXBP5-AS1overexpressing) were prepared into single-cell suspension in PBS $\left(1 \times 10^{6}\right.$ cells $\left./ \mathrm{ml}\right)$, and i.v. injected into the lateral tail vein. After 21 days, all subject mice were sacrificed and lung macro-metastasis was examined with H\&E staining. The animal study was approved by the Institutional Animal Care and Use Committee and in strict accordance with the NIH guideline.

\section{Western blot}

Cells were lysed in RIPA buffer on ice, and protein concentration was quantified by the BCA method (Sigma, MO, USA). $20 \mu \mathrm{g}$ protein was resolved by SDS-PAGE and transferred onto PVDF membrane (Millipore, MA, USA). After brief blocking with $5 \%$ milk, the membrane was probed with primary antibodies: rabbit anti-Sox2 (\#2748, Cell Signaling Technology, MA, USA), rabbit anti-Bmi1 (\#6964, Cell Signaling Technology, MA, USA), rabbit anti-Lin28 (\#3695, Cell Signaling Technology, MA, USA), rabbit anti-Nanog (\#8822, Cell Signaling Technology, MA, USA), rabbit anti-ADGB (ab204085, Abcam, Cambridge, UK), rabbit anti- $\beta$ actin (\#4970, Cell Signaling Technology, MA, USA) at $4{ }^{\circ} \mathrm{C}$ overnight. After washing, membranes were hybridized with secondary antibodies for another hour. The blots were detected with ECL Kit (APPLYGEN, Beijing, China) and visualized on LI-COR system (Biosciences, Lincoln, NE, USA).

\section{Xenograft tumor model}

To evaluate the tumorigenic capacity, PANC-1 cells (control or STXBP5-AS1-overexpression, $2 \times 10^{3}$, $2 \times 10^{4}, 2 \times 10^{5}, 2 \times 10^{6}, 2 \times 10^{7}$ cells) were subcutaneously injected into nude mice ( $n=8$ for each group). Tumor progression was continuously monitored for up to 2 weeks. All mice were then sacrificed, and xenograft tumor formation was validated by pathological examination.

\section{Subcellular localization}

PARIS Kit (Life Technologies, Carlsbad, CA, USA) was employed to fractionize cell nuclear and cytosol RNA. RNA was extracted and reversely transcribed as previously described. The relative distribution of STXBP5$A S 1$ was measured by real-time PCR. GAPDH and $U 6$ were employed as reference for cytosol and nuclear localization, respectively.

\section{RNA immunoprecipitation (RIP)}

RIP assay was used to evaluate binding between STXBP5-AS1 and EZH2. The assay was performed with 
the EZ-Magna RIP Kit (Millipore, MA, USA) following the manufacturer's manual. Anti-EZH2 antibody and control IgG were obtained from Abcam. The immunoprecipitated RNA was recovered and further analyzed by qRT-PCR as previously described.

\section{Chromatin immunoprecipitation (ChIP)}

ChIP was conducted using EZ-Magna ChIP Chromatin Immunoprecipitation Kit (Millipore, MA, USA) according to the manufacturer's recommendation. Chromatin cross-linked with $37 \%$ formaldehyde was ultrasonicated to generate DNA fragments with the average length of 500 to $1000 \mathrm{bp}$. The DNA species were precipitated with EZH2 antibody and recovered, which was further detected and quantified by qRT-PCR.

\section{Bisulfite sequencing PCR (BSP)}

DNA methylation of $A D G B$ promoter was measured with commercially available kits. Genomic DNA from indicated cells was extracted with the DNeasy ${ }^{\circledR}$ Blood and Tissue Kit (Qiagen, CA, USA), which was followed by bisulfate modification. Bisulfite sequencing was then performed with EpiTect ${ }^{\circledR}$ Bisulite Kit (Qiagen, CA, USA) in accordance with the provider's instructions.

\section{Statistical analysis}

Data are reported as mean \pm standard deviation (SD). The inter-group comparison was analyzed using Student's t test or one-way ANOVA analysis with a post hoc test using SPSS 23.0. $P<0.05$ was regarded as statistically significant.

\section{Results}

Decreased STXBP5-AS1 predicted poor prognosis in PC.

First of all, we analyzed a group of 60 PC patients, whose clinical-pathological features are listed in Additional file 1: Table S1. Analysis into these equally grouped patient samples, with respect to $S T X B P 5-A S 1$ transcript levels, manifested favorable both overall and relapse-free survival linking to relatively high STXBP5-AS1 (Fig. 1a, b). Next, STXBP5-AS1 expression in lymph node metastasis (LNM) patients and lymph node metastasis-free (LNMF) patients were significantly decreased compared to respective adjacent normal tissues (ANT) (Fig. 1c). Furthermore, data from GEO datasets showed significant decreased STXBP5-AS1 in PC as compared to the benign tissues as well (Fig. 1d, GSE16515; Fig. 1e, GSE15471). We then determined the relative expression of STXBP5$A S 1$ in a panel of PC cells and found significant downregulation in all tested cancer cell lines in comparison with normal pancreatic duct cell line Htert-HPNE (Fig. 1f). The suppressive expression of STXBP5-AS1 was also noticed in Gemcitabine-resistant (GR) cells compared to parental ones in both PANC-1 and Mia PaCa-2 cell lines (Fig. 1g). The lower abundance of STXBP5-AS1 was especially characterized in sphere derived from these two cells (Fig. 1h), which might imply a possible correlation between down-regulated STXBP5-AS1 and cell stemness. Therefore, our data suggested that downregulation of STXBP5-AS1 in PC might be mechanistically associated with GR and tumor cell stemness, as well as poorer clinical outcome.

\section{STXBP5-AS1 inhibited chemoresistance and metastasis of PC cells.}

Next, we complemented GR cell lines derived from both PANC-1 and Mia PaCa-2 cells with ectopic STXBP5$A S 1$ (Fig. 2a). Drug resistance to Gemcitabine was significantly compromised by $S T X B P 5-A S 1$ as indicated by reduction of $\mathrm{IC}_{50}$ value of Gemcitabine (Fig. 2b). The colony formation capacity of PANC-1/GR and Mia PaCa-2/ GR cells was greatly inhibited by ectopic STXBP6-AS1 (Fig. 2c). Concurrently, activation of capase-3 in response to Gemcitabine exposure was tremendously augmented by over-expression of STXBP5-AS1 in both GR cells (Fig. 2d). Correspondingly, remarkably increased cell apoptosis was observed STXBP5-AS1-proficient cells in comparison with parental ones upon treatment with $50 \mathrm{ng} / \mathrm{ml}$ Gemcitabine (Fig. 2e). In addition to GR cells, we generated STXBP5-AS1-overexpressing cells in naïve PANC-1 and Mia PaCa-2 cells as well (Fig. 2f). Cell invasion was evidently inhibited by ectopic STXBP5-AS1 in both PANC-1 and Mia PaCa-2 cells (Fig. 2g). More importantly, we provided evidences in support of the metastasis-inhibiting effect of STXBP5-AS1 in PANC-1 lung metastasis model. STXBP5-AS1-proficiency greatly suppressed lung metastatic loci establishment of tail vein-injected PANC-1 cells, as shown in the representative $H \& E$ staining of lung sections (Fig. 2h). Statistics suggested that lung metastasis occurred in 7 out 8 of vector control mice, while absent in only 1 out 8 STXBP5-AS1complemented mice (Fig. 2i). Our data showed that STXBP5-AS1 significantly improved chemosensitivity of GR cells, whereas it greatly blockaded metastasis of naïve PC cells.

\section{STXBP5-AS1 suppressed stemness of PC cells}

Our preliminary data showed downregulation of STXBP5-AS1 in PC cell-derived spheres, which hinted the potential causal relation between STXBP5-AS1 and tumor cell stemness. To further clarify this issue, we over-expressed STXBP5-AS1 in both PANC-1 and Mia $\mathrm{PaCa}-2$ cells, and examined the influence of STXBP5-AS1 on sphere formation. As shown in Fig. 3a, the sphere formation capacity was greatly compromised by STXBP5AS1 in both cells. Molecular profiling of cell stemness 


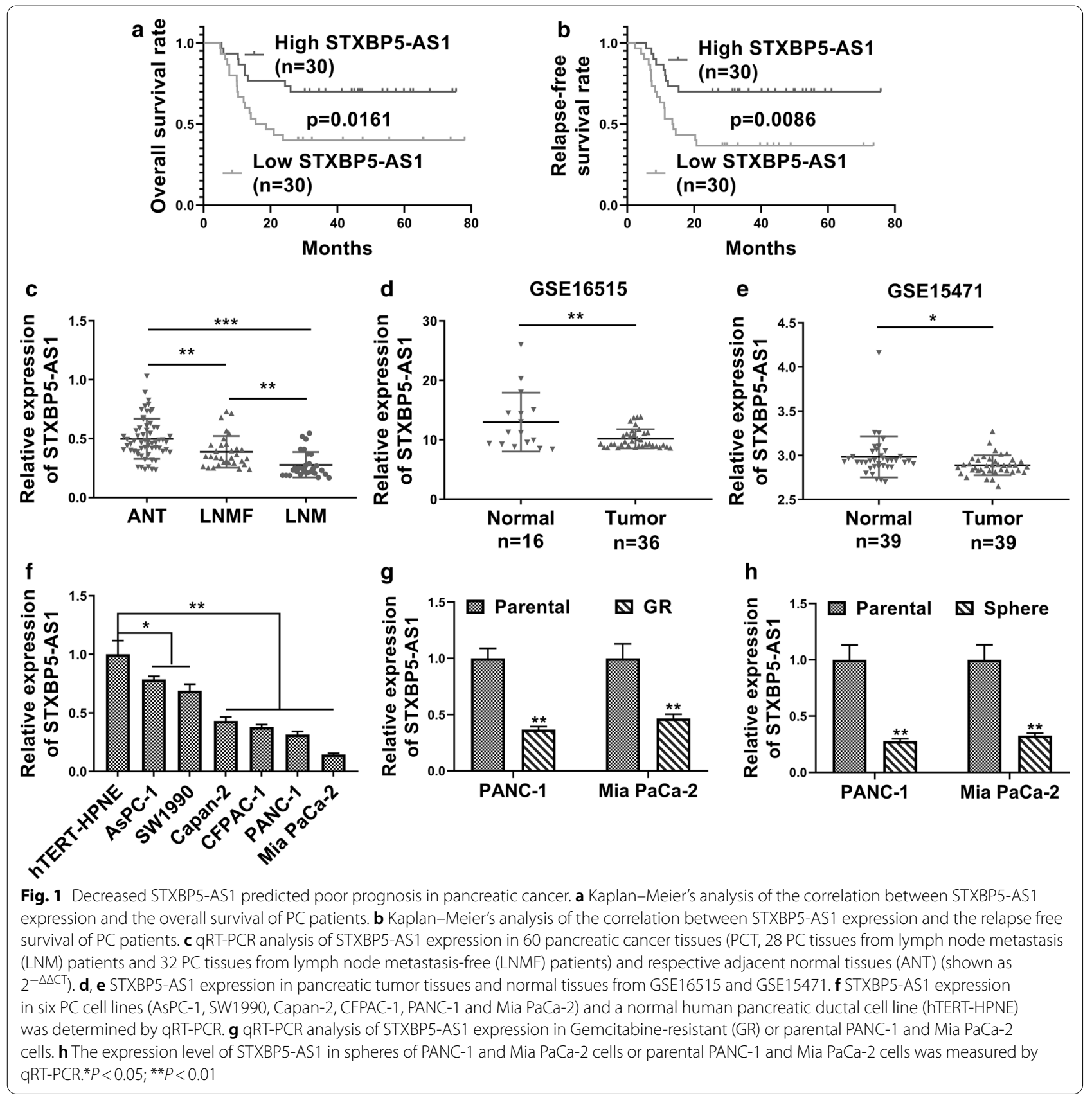

markers including Sox2, Bmi1, Lin28 and Nanog demonstrated remarkable reduction of all of four markers in response to STXBP5-AS1 overexpression in both PANC-1 and Mia PaCa-2 cells at the transcriptional level (Fig. 3b, c). The suppressed expression of Sox2, Bmi1, Lin28 and Nanog was validated at protein level by Western blot analysis (Fig. 3d). Most importantly, compromised stemness by STXBP5-AS1 was demonstrated by limiting dilution assay of xenograft tumor incidence. We noticed that STXBP5-AS1-proficiency greatly inhibited the incidence of PANC-1 cell-derived xenograft tumor, while total injected cell number was limited to $2 \times 10^{6}$ and less (Fig. 3e). Taken together, our data supported the suppressive effects of STXBP5-AS1 on stemness of PC cells both in vitro and in vivo.

\section{STXBP5-AS1 epigenetically regulated neighboring gene $A D G B$ transcription by binding to $E Z H 2$}

Next, we sought to understand the molecular mechanism underlying the tumor-suppressor role of STXBP5-AS1 in 


\section{(See figure on next page.)}

Fig. 2 STXBP5-AS1 inhibited chemoresistance and metastasis of PC cells. a The overexpression efficiency of STXBP5-AS1 in Gemcitabine-resistant PANC-1 and Mia PaCa-2 (PANC-1/GR and Mia PaCa-2/GR) cells transfected with STXBP5-AS1 plasmid (pSin-STXBP5-AS1) or empty vector (pSin-vector) was confirmed by qRT-PCR. $\mathbf{b}$ Determination of the effects of STXBP5-AS1 overexpression on Gemcitabine IC ${ }_{50}$ in PANC-1/GR and Mia PaCa-2/GR cells using MTT assay. c Quantification of colony formation in Gemcitabine-challenged (50 ng/ml) PANC-1/GR and Mia PaCa-2/GR cells transfected with STXBP5-AS1 plasmid (pSin-STXBP5-AS1) or empty vector (pSin-vector). d, e PANC-1/GR and Mia PaCa-2/GR cells transfected with STXBP5-AS1 plasmid (pSin-STXBP5-AS1) or empty vector (pSin-vector) cells were treated with $50 \mathrm{ng} / \mathrm{ml}$ Gemcitabine for $48 \mathrm{~h}$, the apoptosis was analyzed by caspase-3 activity assay and FACS. f The overexpression efficiency of STXBP5-AS1 in PANC-1 and Mia PaCa-2 cells stably transfected with STXBP5-AS1 plasmid (pSin-STXBP5-AS1) or empty vector (pSin-vector) was confirmed by qRT-PCR. $\mathbf{g}$ Transwell assay indicated that STXBP5-AS1 repressed the invasion of PANC-1 and Mia PaCa-2 cells. $\mathbf{h}$ and $\mathbf{i}$ H\&E staining of the metastatic nodules in the lung of PANC-1 cells stably transfected with STXBP5-AS1 plasmid (pSin-STXBP5-AS1) or empty vector (pSin-vector) following tail vein injection into nude mice (200x scale bars) and incidence of lung metastasis in mice following tail vein injection of the respective PANC-1 cells. ${ }^{*} P<0.05$; ${ }^{*} P<0.01$ ( $X^{2}$ test for 1 , Student's $t$ test for others)

PC. We focused on the neighboring genes in view of the well-recognized mode of action of lncRNA in regulating adjacent genes. We found $A D G B$ was greatly inhibited by STXBP5-AS1 in both PANC-1 and Mia PaCa-2 cells (Fig. 4a). In contrast, transcripts of $A D G B$ were markedly up-regulated in STXBP5-AS1-depleted cells (Fig. 4b). The regulatory effects of $S T X B P-A S 1$ on $A D G B$ were further confirmed by Western blot analysis (Fig. 4c). To gain further insight into the regulatory mechanism, we then examined the subcellular localization of STXBP5-AS1 transcripts via fractionation PCR analysis. As suggested by Fig. 4d, e, the majority of STXBP5-AS1 from both PANC-1 and Mia PaCa-2 cells existed in the nuclear fraction with a minor proportion detectable in the cytoplasm, which indicated that STXBP5-AS1 exerted physiological roles predominantly in the nucleus. Multiple lncRNAs have been previously identified to be involved in complex with $E Z H 2$ and therefore in regulation of promoter methylation of target genes. Along this direction, we detected the enrichment of STXBP5-AS1 transcripts in $E Z H 2$ immunoprecipitated RNA species in both PANC-1 and Mia PaCa-2 cells (Fig. 4f). Meanwhile, direct association of EZH2 with $A D G B$ promoter was demonstrated by ChIP assay as shown in Fig. 4g, which implicated the role of PRC2 complex in the epigenetic regulation of $A D G B$. The relative enrichment of $A D G B$ promoter was significantly decreased by siRNA-mediated knockdown of STXBP5-AS1 in comparison with scramble control (Fig. 4h, i). The association of EZH2 with $A D G B$ promoter therefore was greatly dependent on STXBP5-AS1. Consistently, over-expression of STXBP5-AS1 increased the enrichment of $A D G B$ promoter in EZH2 immunoprecipitated complex, which suggested an enhancement of EZH2 binding to $A D G B$ promoter (Fig. 4j, k). Consequently, methylation level of $A D G B$ promoter region was tremendously decreased in response to STXBP5AS1 knockdown, which was comparable with treatment by the DNA demethylating agent 5-Aza-CdR (Fig. 4l). STXBP5-AS1 overexpression oppositely increased methylation status of $A D G B$ promoter, which was readily abrogated by simultaneous EZH2-knockdown or 5-AzaCdR treatment (Fig. 4m). Summarily, we provided evidence that STXBP5-AS1 potently inhibited expression of neighboring $A D G B$ via an epigenetic mechanism, specifically through complexation with EZH2.

\section{STXBP5-AS1 inhibited stem cell-like properties of PC cells by suppressing $A D G B$ expression}

Next, we sought to clarify whether down-regulated $A D G B$ mainly mediated the inhibitory effects of STXBP5-AS1 on cell stemness in PC cells. To this end, we established STXBP5-AS1-overexpressing and ADGBoverexpressing cells either individually or in combination in parental PANC-1 and Mia PaCa-2 cells (Fig. 5a, b), as well as in the respective GR cells (Fig. 5c, d). Consistent with previous observation, forced expression of STXBP5-AS1 greatly improved the sensitivity of GR cells, which was almost completely reversed by co-expression of $A D G B$ (Fig. 5e). Likewise, colony formation capacity was compromised in response to ectopic STXBP5-AS1 in both PANC-1/GR and Mia PaCa-2/GR cells, while restored by complementation of $A D G B$ (Fig. 5f). Caspase-3 activation and apoptotic index, which were greatly stimulated by introduction of STXBP5-AS1, were inhibited by $A D G B$ overexpression as well (Fig. $5 \mathrm{~g}, \mathrm{~h}$ ). The invasive capacity compromised by $S T X B P 5-A S 1$ was greatly recovered by simultaneous expression of $A D G B$ (Fig. 5i). Similarly, $A D G B$ complementation evidently restored the colony formation in STXBP5-AS1-proficient cells (Fig. 5j). At the molecular level, co-overexpression of $A D G B$ in the context of ectopic STXBP5-AS1 expression up-regulated Sox2, Bim1, Lin28 and Nanog, which was significantly inhibited by STXBP5-AS1-overexpression alone (Fig. $5 \mathrm{k}, \mathrm{l}$ ). This change was also validated at the protein level by Western blot analysis (Fig. $5 \mathrm{~m}$ ). Therefore, our results supported that STXBP5-AS1 decreased the stem cell-like properties of PC cells mainly by epigenetic suppression of $A D G B$. 

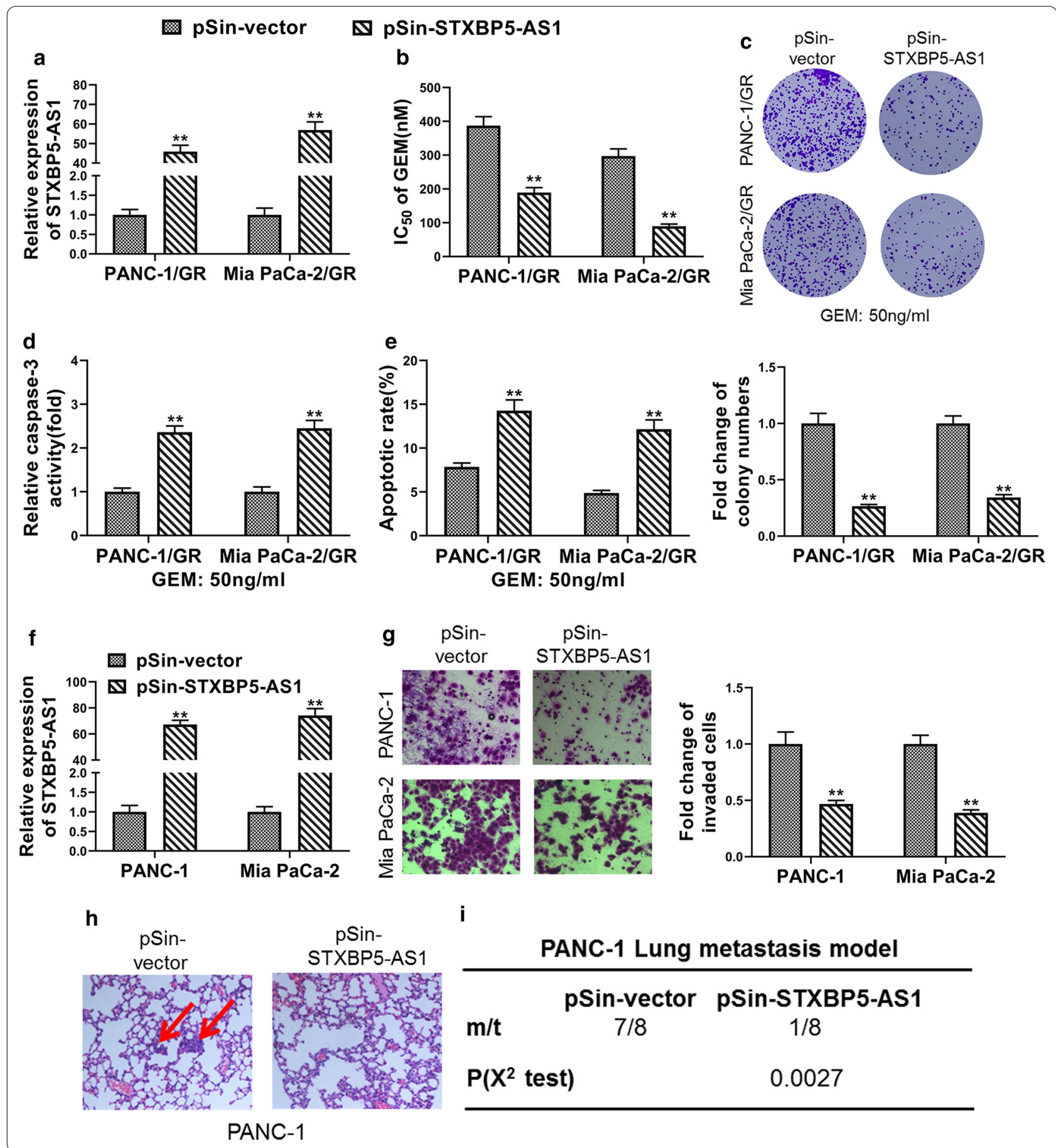

PANC-1 Lung metastasis model

\begin{tabular}{lcc} 
& pSin-vector \\
$\mathrm{m} / \mathrm{t}$ & $\mathrm{pSin-STXBP5-AS1}$ \\
$\mathrm{P}\left(\mathrm{X}^{2}\right.$ test $)$ & & $1 / 8$ \\
\hline
\end{tabular}

\section{Discussion}

Despite previously reported tumor suppressor roles in cervical cancer, gastric cancer and non-small-cell lung cancer $[19,21]$, the relative expression pattern and functional mechanism of STXBP5-AS1 in PC were still obscure currently. Here, we first characterized aberrant downregulation of STXBP5-AS1 in PC both in vitro and in vivo. Particularly, a potential association was observed between STXBP5-AS1 deficiency and cell stemness and drug resistance. In addition, high expression of STXBP5-AS1 was significantly enriched in the PC patients without LNM, and consequently associated with both overall and relapse-free survival clinically. In cell culture, overexpression of STXBP5-AS1 rendered 

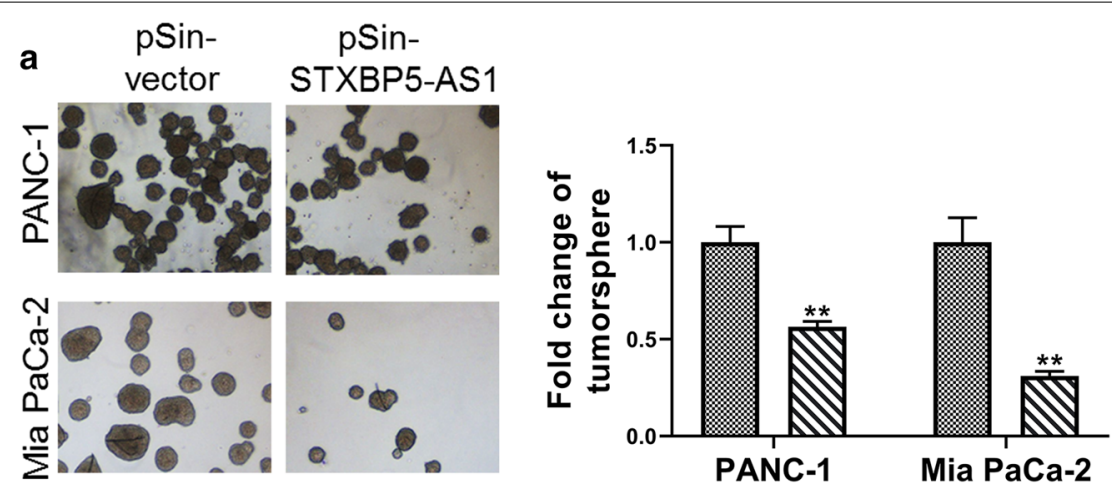

b

PANC-1

C

Mia PaCa-2
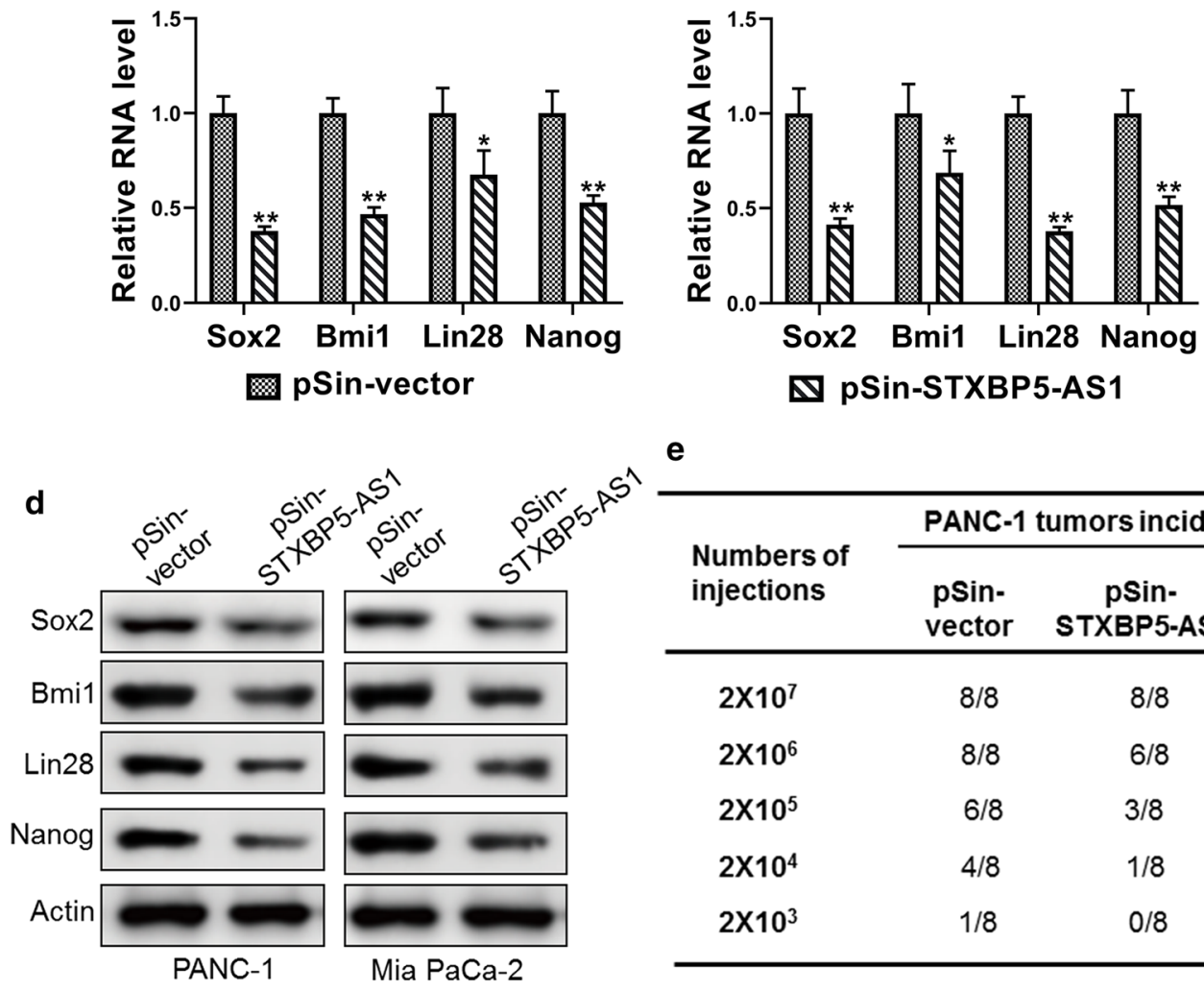

e

\begin{tabular}{ccc}
\multirow{2}{*}{$\begin{array}{l}\text { Numbers of } \\
\text { injections }\end{array}$} & \multicolumn{2}{c}{ PANC-1 tumors incidence } \\
\cline { 2 - 3 } & $\begin{array}{c}\text { pSin- } \\
\text { vector }\end{array}$ & $\begin{array}{c}\text { pSin- } \\
\text { STXBP5-AS1 }\end{array}$ \\
\hline $2 \times 10^{7}$ & $8 / 8$ & $8 / 8$ \\
$2 \times 10^{6}$ & $8 / 8$ & $6 / 8$ \\
$2 \times 10^{5}$ & $6 / 8$ & $3 / 8$ \\
$2 \times 10^{4}$ & $4 / 8$ & $1 / 8$ \\
$2 \times 10^{3}$ & $1 / 8$ & $0 / 8$ \\
\hline
\end{tabular}

Fig. 3 STXBP5-AS1 suppressed stemness of PC cells. a Sphere formation of PANC-1 and Mia PaCa-2 cells stably transfected with STXBP5-AS1 plasmid (pSin-STXBP5-AS1) or empty vector (pSin-vector). The total sphere numbers in each well were counted and images were taken at 40x magnification. b-d The expression levels of stem markers (Sox2, Bmi1, Lin28 and Nanog) in PANC-1 and Mia PaCa-2 cells stably transfected with STXBP5-AS1 plasmid (pSin-STXBP5-AS1) or empty vector (pSin-vector) were assessed by qRT-PCR and western blot. e Tumor incidence of PANC-1 cells stably transfected with STXBP5-AS1 plasmid (pSin-STXBP5-AS1) or empty vector (pSin-vector); cells were injected into the flank of mice with limiting dilutions as indicated. The number of tumors formed in each group was counted after 4 weeks. ${ }^{*} P<0.05$; ${ }^{*} P<0.01$

GR cells sensitivity to Gemcitabine and greatly inhibited the colony formation capacity, which was accompanied with caspase-3 activation and cell apoptosis induction. In parental PC cells, the invasive behavior was suppressed by ectopic STXBP5-AS1. This phenotype was validated in vivo with tail vein injection of both empty control and STXBP5-AS1-overexpressing
PANC-1 cells into nude mice as well. Notably, we observed the sphere formation efficacy was significantly compromised by STXBP5-AS1 with concurrent downregulation of stemness markers including Sox2, Bim1, Lin 28 and Nanog. The suppressed stem cell-like properties were especially validated by the limiting dilution of xenograft tumor incidence. Mechanistically, we showed 


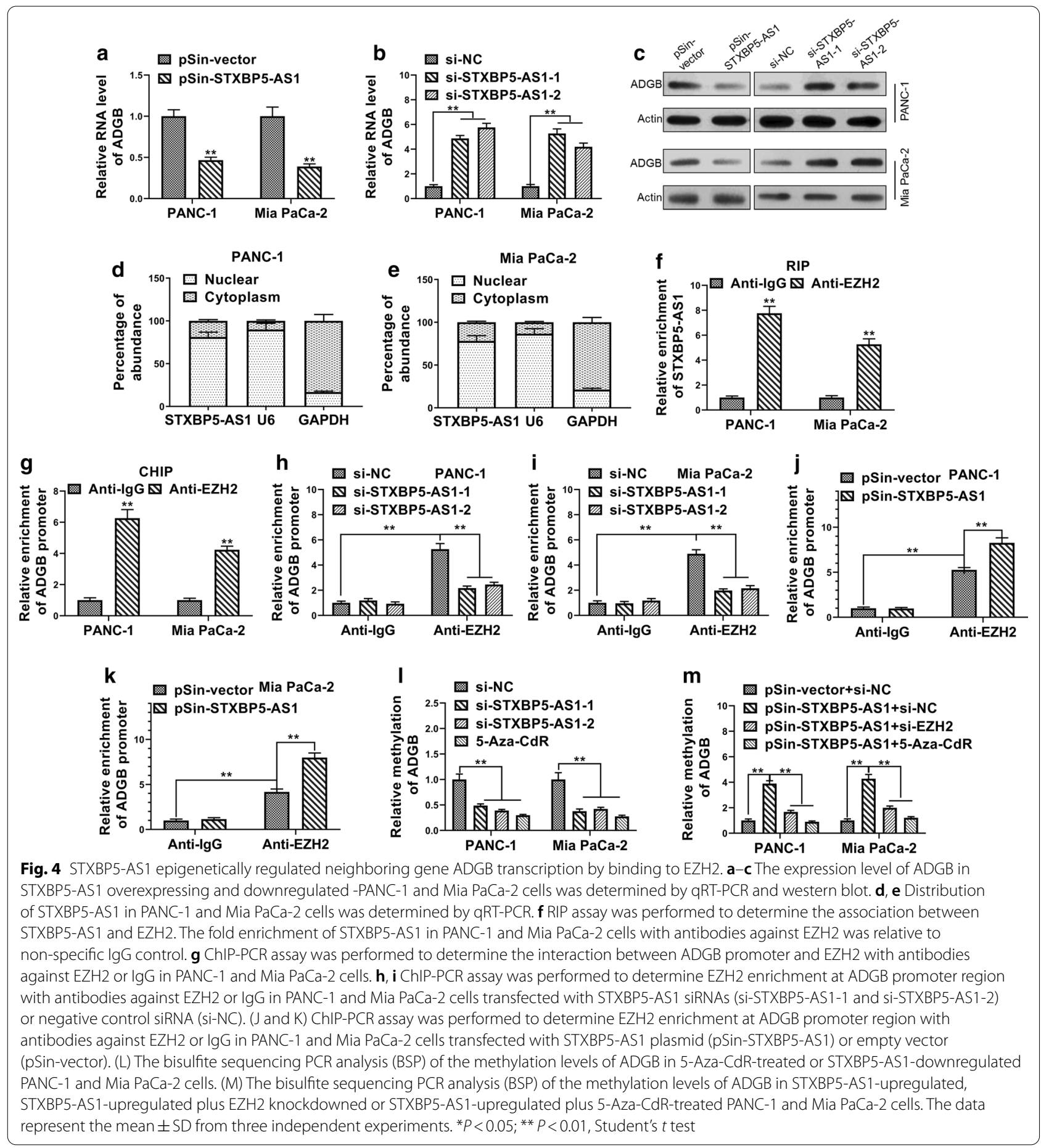

STXBP5-AS1 epigenetically inhibited neighboring $A D G B$ expression through potently recruiting EZH2 to the $A D G B$ promoter and therefore enhancing DNA methylation. Complementation with $A D G B$ remarkably restored drug resistance and colony formation in STXBP5-AS1-proficient GR cells, and simultaneously suppressed apoptotic activation. In parental PC cells, $A D G B$ overexpression rescued the compromised cell invasion and decreased cell stemness markers. In summary, we provided experimental data supporting the tumor suppressor function of STXBP5-AS1 in $\mathrm{PC}$ via inhibiting chemoresistance and stem cell-like 


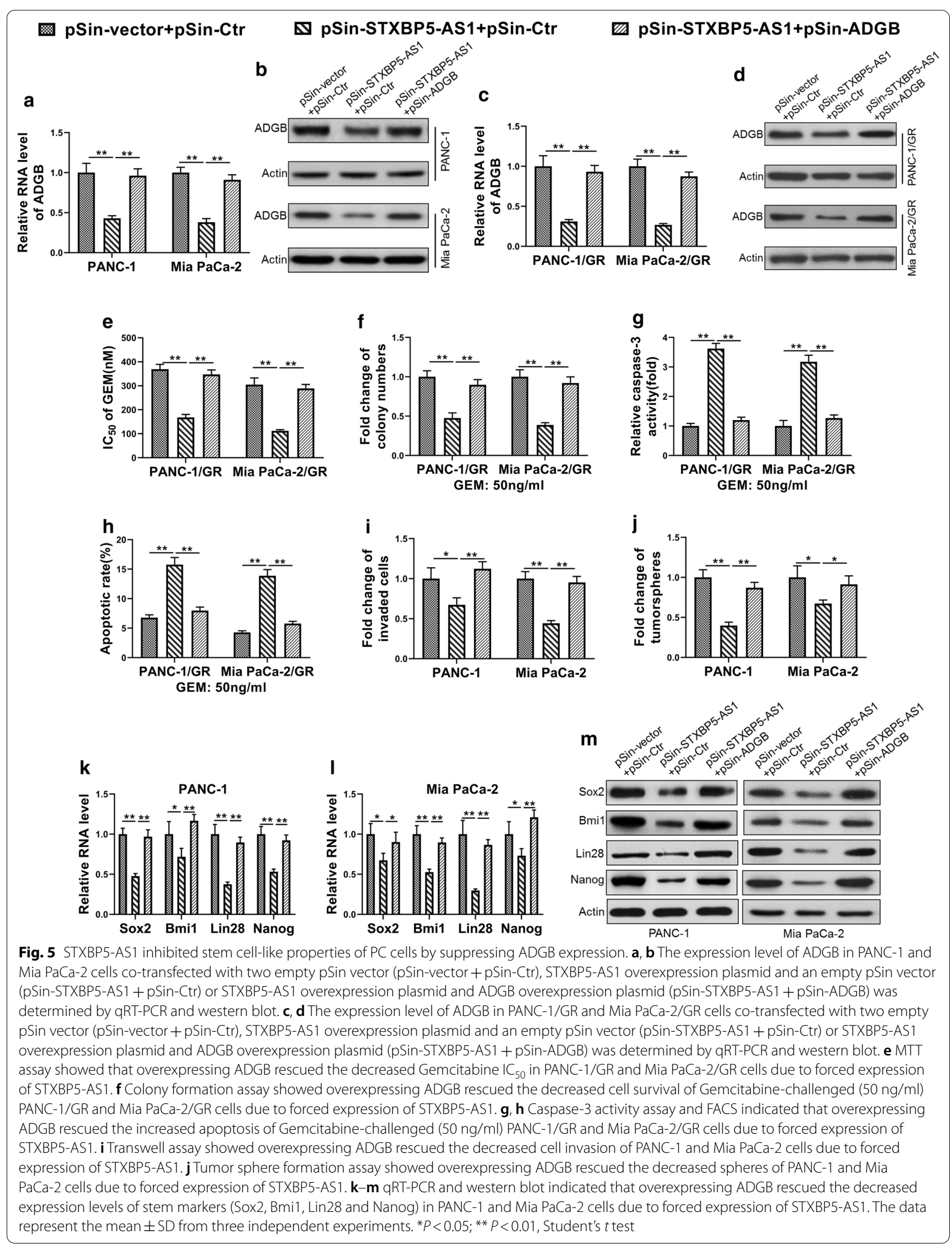


properties, which was greatly mediated by epigenetic silencing of its neighboring $A D G B$.

Emerging evidences have suggested the involvement of IncRNAs in complexation with EZH2 and exerted epigenetic regulation in human cancers. Our data confirmed this notion via demonstrating the direct interaction between STXBP5-AS1 and EZH2, which specified recruitment of EZH2 to the $A D G B$ promoter and led to intensive DNA methylation and gene expression silencing. Our observations resembled multiple scenarios previously described. For instance, Liu et al. reported that LINC01088 enhanced cell proliferation through scaffolding EZH2 and inhibiting p21 in human non-smallcell lung cancer [23]. Song et al. showed that LINC01535 induced cervical cancer progression by specific targeting the miR-214/EZH2 feedback loop [24]. In colorectal cancer, Di et al. demonstrated that SNHG14 facilitated distal metastasis via regulating EZH2-targeted EPHA1 [25]. Xu et al. suggested FOXD2-AS1 functioned as an oncogene in hepatocellular carcinoma by epigenetic suppression of CDKN1B (p27) with EZH2 [26]. Although we exhibited the direct association between STXBP5-AS1 with EZH2, the detailed complexation was still to be elaborated by structural analysis.

ADGB (androglobin) was firstly described as a chimeric globin in metazoans and preferentially expressed in mammalian testis with poorly understood physiologic functions [27]. Until recently, the study performed by Huang et al. proposed that $A D G B$ knockdown in glioma cell lines significantly inhibited cell proliferation and stimulated apoptosis [28], suggesting the potential oncogenic properties of $A D G B$ in this disease. Consistent with this work, our data exhibited that overexpression of $A D G B$ in STXBP5-AS1-proficient GR cells greatly stimulated drug resistance and inhibited cell apoptosis, while rendered stem cell-like properties and invasive potential to parental PC cells in the context of STXBP5-AS1overexpression. Notably, the intensive methylation was detected in the promoter region of $A D G B$ indicated the possible efficacy of DNA demethylating agents for therapeutic purpose, which is definitely worthy of further investigations in the near future. In view of the critical contributions of $A D G B$ to drug resistance of $\mathrm{PC}$, we also raised the hypothesis that combinational administration of both Gemcitabine and 5-Aza-CdR would greatly benefit the intrinsic resistant patients clinically.

Noteworthily, despite the significant downregulation of STXBP5-AS1 characterized here in PC along with previous reports in both cervical and gastric cancers, the molecular mechanisms underlying this phenotype were still elusive currently. Another study limitation is that not all the clinical information was available in the public databases; therefore, the association of STXBP5-AS1 with survival cannot be analyzed. In the future, we would focus on this issue with the aid of bioinformatic analysis of publicly available tumor genome databases. In summary, we demonstrated that the STXBP5-AS1/EZH2/I axis in $\mathrm{PC}$ contributed to the chemoresistance and stem cell-like features.

\section{Supplementary information}

Supplementary information accompanies this paper at https://doi. org/10.1186/s13148-020-00961-y.

Additional file 1: Table S1. Correlation of STXBP5-AS1 expression with clinicopathological features in 60 pancreatic cancer patients.

\section{Abbreviations}

LncRNA: Long non-coding RNA; PC: Pancreatic cancer; GR: Gemcitabine-resistant; RIP: RNA-immunoprecipitation; ChIP: Chromatin immunoprecipitation.

\section{Acknowledgements}

None.

\section{Authors' contributions}

Shi Chen, Long Huang, Ge Li, Funan Qiu, Yaodong Wang, Can Yang, Jingjing Pan, Zhangwei Wu conducted the experiments. Shi Chen, Jiangzhi Chen and Yifeng Tian prepared the main text with help of Long Huang, Ge Li, Funan Qiu and Yaodong Wang. Can Yang, Jingjing Pan and Zhangwei Wu prepared the figures. Shi Chen, Jiangzhi Chen and Yifeng Tian wrote the manuscript. All authors contributed to the content. All authors read and approved the final manuscript.

\section{Funding}

The study was supported by the High-level hospital foster grants from Fujian Provincial Hospital (2019HSJJ13); the Natural Science Foundation for Distinguished Young Scholars of Fujian Province (2018J06020); Education and Scientific Research Foundation of Fujian Province (2060402); Joint Funds for the innovation of science and technology, Fujian Province (2018Y9098, 2018Y9013); Fujian Provincial Health and Family Planning Research Medical Innovation Project (2019-cx-3); the National Natural Science Foundation of China (81772560, 81702438); Youth Project Foundation of Fujian Provincial Health Commission (2018-1-10); Fujian Provincial Health and Family Planning Research Talents Cultivation Project (2017-ZQN-27).

Availability of data and materials

Not applicable.

\section{Ethics approval and consent to participate}

The study was approved by the ethics commitment of Fujian Provincial Hospital, Shengli Clinical Medical College of Fujian Medical University, Fujian Medical University.

\section{Competing interests}

The authors declare that they have no competing interests.

\section{Consent for publication}

Not applicable.

Received: 10 July 2020 Accepted: 27 October 2020

Published online: 07 November 2020

\section{References}

1. Jemal A, Bray F, Center MM, Ferlay J, Ward E, Forman D. Global cancer statistics. CA Cancer J Clin. 2011;61:69-90. 
2. Fogel EL, Shahda S, Sandrasegaran K, DeWitt J, Easler JJ, Agarwal DM, Eagleson M, Zyromski NJ, House MG, Ellsworth S, et al. A multidisciplinary approach to pancreas cancer in 2016: a review. Am J Gastroenterol. 2017;112:537-54.

3. Edwards BK, Brown ML, Wingo PA, Howe HL, Ward E, Ries LA, Schrag D, Jamison PM, Jemal A, Wu XC, et al. Annual report to the nation on the status of cancer, 1975-2002, featuring population-based trends in cancer treatment. J Natl Cancer Inst. 2005;97:1407-27.

4. Arslan AA, Helzlsouer KJ, Kooperberg C, Shu XO, Steplowski E, Bueno-deMesquita HB, Fuchs CS, Gross MD, Jacobs EJ, Lacroix AZ, et al. Anthropometric measures, body mass index, and pancreatic cancer: a pooled analysis from the Pancreatic Cancer Cohort Consortium (PanScan). Arch Intern Med. 2010;170:791-802.

5. Hermann PC, Huber SL, Heeschen C. Metastatic cancer stem cells: a new target for anti-cancer therapy? Cell Cycle. 2008;7:188-93.

6. Hermann PC, Huber SL, Herrler T, Aicher A, Ellwart JW, Guba M, Bruns CJ, Heeschen C. Distinct populations of cancer stem cells determine tumor growth and metastatic activity in human pancreatic cancer. Cell Stem Cell. 2007;1:313-23.

7. Li C, Heidt DG, Dalerba P, Burant CF, Zhang L, Adsay V, Wicha M, Clarke MF, Simeone DM. Identification of pancreatic cancer stem cells. Cancer Res. 2007:67:1030-7.

8. Bar EE, Chaudhry A, Lin A, Fan X, Schreck K, Matsui W, Piccirillo S, Vescovi AL, DiMeco F, Olivi A, Eberhart CG. Cyclopamine-mediated hedgehog pathway inhibition depletes stem-like cancer cells in glioblastoma. Stem Cells. 2007;25:2524-33.

9. Mueller MT, Hermann PC, Witthauer J, Rubio-Viqueira B, Leicht SF, Huber S, Ellwart JW, Mustafa M, Bartenstein P, D'Haese JG, et al. Combined targeted treatment to eliminate tumorigenic cancer stem cells in human pancreatic cancer. Gastroenterology. 2009;137:1102-13.

10. Liu L, Yin S, Brobbey C, Gan W. Ubiquitination in cancer stem cell: roles and targeted cancer therapy. STEMedicine. 2020;1:e37.

11. Quinn JJ, Chang HY. Unique features of long non-coding RNA biogenesis and function. Nat Rev Genet. 2016;17:47-62.

12. Jiang $\mathrm{Q}$, Wang J, Wu X, Ma R, Zhang T, Jin S, Han Z, Tan R, Peng J, Liu G, et al. LncRNA2Target: a database for differentially expressed genes after IncRNA knockdown or overexpression. Nucleic Acids Res. 2015;43:D193-196.

13. Paraskevopoulou MD, Hatzigeorgiou AG. Analyzing MiRNA-LncRNA interactions. Methods Mol Biol. 2016;1402:271-86.

14. Xu CZ, Jiang C, Wu Q, Liu L, Yan X, Shi R. A feed-forward regulatory loop between HuR and the long noncoding RNA HOTAIR promotes head and neck squamous cell carcinoma progression and metastasis. Cell Physiol Biochem. 2016;40:1039-51.

15. Heery R, Finn SP, Cuffe S, Gray SG. Long non-coding RNAs: key regulators of epithelial-mesenchymal transition, tumour drug resistance and cancer stem cells. Cancers (Basel) 2017, 9.
16. Tsai MC, Manor O, Wan Y, Mosammaparast N, Wang JK, Lan F, Shi Y, Segal E, Chang HY. Long noncoding RNA as modular scaffold of histone modification complexes. Science. 2010;329:689-93.

17. Lee JT. Epigenetic regulation by long noncoding RNAs. Science. 2012:338:1435-9.

18. Margueron R, Reinberg D. The polycomb complex PRC2 and its mark in life. Nature. 2011:469:343-9.

19. Huang J, Xie N, Huang H, Yao J, Hu W: Long noncoding RNA STXBP5-AS1 inhibits cell proliferation, migration, and invasion via preventing the PI3K AKT against STXBP5 expression in non-small-cell lung carcinoma. J Cell Biochem. 2018

20. Cen D, Huang H, Yang L, Guo K, Zhang J. Long noncoding RNA STXBP5AS1 inhibits cell proliferation, migration, and invasion through inhibiting the PI3K/AKT signaling pathway in gastric cancer cells. Onco Targets Ther. 2019;12:1929-36.

21. Shao $S$, Wang $C$, Wang $S$, Zhang H, Zhang Y. LncRNA STXBP5-AS1 suppressed cervical cancer progression via targeting miR-96-5p/PTEN axis. Biomed Pharmacother. 2019;117:109082.

22. Ham J, Jeong D, Park S, Kim HW, Kim H, Kim SJ. Ginsenoside Rg3 and Korean Red Ginseng extract epigenetically regulate the tumor-related long noncoding RNAs RFX3-AS1 and STXBP5-AS1. J Ginseng Res. 2019:43:625-34.

23. Liu JQ, Feng YH, Zeng S, Zhong MZ. linc01088 promotes cell proliferation by scaffolding EZH2 and repressing p21 in human non-small cell lung cancer. Life Sci. 2020;241:117134.

24. Song H, Liu Y, Jin X, Liu Y, Yang Y, Li L, Wang X, Li G. Long non-coding RNA LINC01535 promotes cervical cancer progression via targeting the miR214/EZH2 feedback loop. J Cell Mol Med. 2019;23:6098-111.

25. Di W, Weinan X, Xin L, Zhiwei Y, Xinyue G, Jinxue T, Mingqi L. Long noncoding RNA SNHG14 facilitates colorectal cancer metastasis through targeting EZH2-regulated EPHA7. Cell Death Dis. 2019;10:514.

26. Xu K, Zhang Z, Qian J, Wang S, Yin S, Xie H, Zhou L, Zheng S. LncRNA FOXD2-AS1 plays an oncogenic role in hepatocellular carcinoma through epigenetically silencing CDKN1B(p27) via EZH2. Exp Cell Res. 2019;380:198-204.

27. Hoogewijs D, Ebner B, Germani F, Hoffmann FG, Fabrizius A, Moens L, Burmester T, Dewilde S, Storz JF, Vinogradov SN, Hankeln T. Androglobin: a chimeric globin in metazoans that is preferentially expressed in Mammalian testes. Mol Biol Evol. 2012;29:1105-14.

28. Huang B, Lu YS, Li X, Zhu ZC, Li K, Liu JW, Zheng J, Hu ZL. Androglobin knockdown inhibits growth of glioma cell lines. Int J Clin Exp Pathol. 2014;7:2179-84.

\section{Publisher's Note}

Springer Nature remains neutral with regard to jurisdictional claims in published maps and institutional affiliations.
Ready to submit your research? Choose BMC and benefit from:

- fast, convenient online submission

- thorough peer review by experienced researchers in your field

- rapid publication on acceptance

- support for research data, including large and complex data types

- gold Open Access which fosters wider collaboration and increased citations

- maximum visibility for your research: over 100M website views per year

At BMC, research is always in progress.

Learn more biomedcentral.com/submissions 